\title{
Recycler transverse instability in context of Proton Plan II
}

\author{
V.Balbekov \\ Fermi National Accelerator Laboratory \\ P.O. Box 500, Batavia, Illinois 60510
}

January 2, 2007

\begin{abstract}
Transverse instability of a bunched beam in a ring accelerator is considered. An emphasis is given to an investigation of rigid modes at space charge dominating beam coupling impedance and about linear synchrotron oscillations. The theory is applied to the Fermilab Recycler [1] which is treated as a proton accumulator in context of Proton Plan II [2]. It is shown that the instability growth time can reach about $0.3 \mathrm{~ms}$, and chromaticity about -7 to -10 is required to suppress it. The suppression by means of $26 \mathrm{MHz}$ damper is considered also, and required parameters of the damper are provided. Arguments are adduced that obtained stability conditions are not only necessary but also sufficient, because other modes except the rigid ones are more stable.
\end{abstract}

\section{Introduction}

It was proposed to use the Fermilab Recycler Ring as a high intensity proton accumulator in a multistage complex known as the Proton Plan [2]. According this, it is planned to accumulate in the Recycler about $5.3 \times 10^{13}$ protons in second stage of the Plan, an intensity which exceeds achieved number of 
antiprotons more than by order of magnitude. Another essential modification is $53 \mathrm{MHz}$ sinusoidal accelerating field instead of existing barrier bucket $\mathrm{RF}$.

One of the problems arising is transverse instability of the high intensity beam. At present, resistive wall instability of antiprotons is observed in the Recycler, and the digital damper is used to suppress it [3] . However, new conditions are so diverged that both characteristics of the instability and requirements to the damper should be examined from the outset.

One circumstance will remain unchanged: transverse space charge beam coupling impedance dominates in the Recycler, significantly exceeding resistive wall and other contributions. Indeed, the Recycler vacuum chamber is stainless steel elliptic pipe of semi-axes $b_{x}=4.8 \mathrm{~cm}, b_{y}=2.2 \mathrm{~cm}$ with the wall thickness $d=1.5 \mathrm{~mm}$ and resistivity $\rho=74 \mu \mathrm{Ohm}-\mathrm{cm}$. At frequency $\omega$, its transverse impedance can be calculated by the formula:

$$
\begin{gathered}
Z(\omega)=\frac{(s-i) Z_{0} R_{0} \delta_{\omega} g}{b_{y}^{3}} \operatorname{coth}\left(\frac{(1-i s) d}{\delta_{\omega}}\right) \\
=(s-i) Z^{(r w)} \sqrt{\left|\frac{\Omega_{0}}{\omega}\right|} \operatorname{coth}\left((1-i s) \sqrt{\left|\frac{\omega}{\Omega_{1}}\right|}\right)
\end{gathered}
$$

where $s=\operatorname{sgn}(\omega), \delta_{\omega}$ is skin depth at frequency $\omega, Z_{0}=4 \pi / c=376.7 \mathrm{Ohm}$, $R_{0}=528.3 \mathrm{~m}$ is the accelerator average radius, $\Omega_{0}=2 \pi \times 89.9 \mathrm{kHz}$ is revolution frequency, and $\Omega_{1}=2 \pi \times 83 \mathrm{kHz}$ (the wall thickness coincides with skin depth at this frequency). Factor $g$ depends on $b_{y} / b_{x}$ ratio, and it is in the Recycler: 0.826 for vertical oscillations and 0.415 for horizontal ones (see Appendix for details). Correspondingly, characteristic impedances $Z^{(r w)}$ are 22 and $11 \mathrm{MOhm} / \mathrm{m}$. At betatron tune $Q_{0}=24.415$, the vertical impedance is about $38 \mathrm{MOhm} / \mathrm{m}$ for the lowest unstable harmonic $\omega=0.585 \Omega_{0}$.

Space charge impedance is associated with betatron tune shift and does not depend on frequency. In particular, for Gaussian beam it is:

$$
Z^{(s c)}=\frac{i Z_{0} Q_{0}}{4 B \beta \gamma \varepsilon}
$$

where $B$ is bunch-factor, $\beta \gamma \simeq 9.47$ is normalized momentum, and $\varepsilon$ is normalized transverse rms beam emittance which is presumed here to be the same both in $x$ and $y$ directions [4]. At present, $\varepsilon \simeq 2 \mathrm{~mm}-\mathrm{mrad}$, and its increase is not foreseen in the Proton Plan because it would cause a surplus 
particle loss. Taking a reasonable value $B \simeq 0.5$, one obtains $Z^{(s c)} \simeq$ $240 i \mathrm{MOhm} / \mathrm{m}$ that is in order of magnitude more of the resistive wall part.

Theory of transverse instability at dominating space charge impedance is not sufficiently advanced at present. The problem was considered first in Ref. [5] where it was shown that the space charge suppresses instability of all modes, except those not depending on synchrotron phase. However, approximation of uncoupled synchrotron multipoles was used in that paper, a method which is applicable if the synchrotron tune is much more than the betatron space charge tune shift. The opposite situation is expected in the Recycler at designed proton intensity. There is a statement in work [6] that the space charge suppresses the fast head-tail instability at these conditions. However, the conclusion was made by analysis of short-range wake fields, an assumption which excluded both bunch-to-bunch and turn-by-turn interactions. Meanwhile, long range interaction produced by the resistive wall is the main instability factor in the Recycler, and collective mode bunched beam instability is the most expected effect. Therefore, the problem requires additional investigations to apply the results to the Recycler.

It is necessary to note in this connection that, in any case, there are the modes of bunched beam oscillations which are not affected by space charge at all, and therefore cannot be suppressed by this manner ${ }^{1}$. These modes are known popularly as "rigid", because the bunches behave in some respects like solid. Motion of their centers does not depend on inter-particle interactions, a category which includes space charge forces as well ${ }^{2}$. Analysis of these modes is the main objective of this note. Its suppression is a necessary condition of the beam stability, and it might be sufficient condition as well, because it looks very probably that these modes are most unstable in practice.

\footnotetext{
${ }^{1}$ It does not mean that space charge is negligible at this consideration. Quite the reverse, it separates the rigid modes by an influence on other ones. The problem is discussed in Sec. 7 .

${ }^{2}$ In fact, the case of points should be groups of particles with the some longitudinal coordinate. Different parts of a bunch can move relative to each other, that is the bunch is not quite "rigid". We do not emphasize it here because it does not change the conclusion. Related details will be discussed in Sec. 4 .
} 


\section{Basic equations}

We are starting from equation of betatron oscillations:

$$
\frac{d^{2} x}{d t^{2}}+\Omega^{2} Q^{2} x=\frac{e E\left(\theta, x-X_{c}(t, \theta)\right)}{m \gamma^{3}}+\frac{e G(t, \theta)}{m \gamma}
$$

where $\Omega$ and $Q$ are the particle angular velocity and betatron tune, $E$ is the space charge electric field, and a small addition $e G$ is Lorenz force per particle due to any other impedance (resistive wall, cavities, etc.). Nonlinearity of external field is neglected here because it is very small in the Recycler. However, linearity of the beam field $E$ is not presumed, that is the space charge density in the beam cross section is not considered as a constant. It is clear that this field depends on deviation of the particle from the bunch center. There is a longitudinal deviation $\theta$, and transverse one $x-X_{c}(t, \theta)$ where $X_{c}(t, \theta)$ is the beam center position at azimuth $\theta$, which can be non-zero because of coherent oscillations. It is presumed also that the force $e G(t, \theta)$ does not depend on transverse coordinate of the particle, and is dictated by the beam center displacement over all the ring.

Let us consider all the particles located in a small region of the longitudinal phase space near the point $(\theta, u)$, where the variable $u$ is proportional to a deviation of the particle momentum from central value (coefficient of the proportionality will be defined later). We will assume that transverse distribution of these particles is described by the normalized function $\rho_{\perp}(x-X(t, \theta, u))$ where $X(t, \theta, u)$ is position of the group center, that is

$$
X(t, \theta, u)=\int_{-\infty}^{\infty} \rho_{\perp}(x-X(t, \theta, u)) x d x=\int_{-\infty}^{\infty} \rho_{\perp}(x)[x+X(t, \theta, u)] d x
$$

In accordance with Eq. (3), this function satisfies the equation:

$$
\begin{gathered}
{\left[\frac{d^{2}}{d t^{2}}+\Omega^{2}(u) Q^{2}(u)\right] X(t, \theta, u)=} \\
\frac{e}{m \gamma^{3}} \int_{-\infty}^{\infty} E\left(\theta, x+X(t, \theta, u)-X_{c}(t, \theta)\right) \rho_{\perp}(x) d x+\frac{e G(t, \theta)}{m \gamma}
\end{gathered}
$$

where an important thing is marked that the revolution frequency and the tune can depend on the particle momentum. Note also that $\rho_{\perp}(x)$ and 
$E(\theta, x)$ are, respectively, even and odd functions of $x$. Therefore, assuming that both $X$ and $X_{c}$ are small in comparison with the beam transverse dimension, it is possible to rewrite Eq. (5) in the form:

$$
\begin{gathered}
{\left[\frac{d^{2}}{d t^{2}}+\Omega^{2}(u) Q^{2}(u)\right] X(t, \theta, u) \simeq} \\
2 \Omega_{0}^{2} Q_{0} \Delta Q(\theta)\left[X(t, \theta, u)-X_{c}(t, \theta)\right]+\frac{e G(t, \theta)}{m \gamma}
\end{gathered}
$$

where $\Omega_{0}$ and $Q_{0}$ are the central values of the variables, and

$$
\Omega_{0}^{2} Q_{0} \Delta Q(\theta)=\frac{e}{2 m \gamma^{3}} \int_{-\infty}^{\infty} \frac{\partial E}{\partial x}(\theta, x) \rho_{\perp}(x) d x .
$$

As it is shown in Ref. [4], $\Delta Q(\theta)$ is a positive value numerically coinciding with betatron tune shift driven by space charge and averaged over transverse coordinates. It coincides with usual tune shift if the beam has an elliptical cross section at constant space charge density. For Gaussian beam, the average value is 2 times less than tune shift of small betatron oscillations.

Now we need to take into account that $d / d t$ is actually total derivative over time where the longitudinal motion should be taken into account. In the bunch rest frame it can be written in the form:

$$
\frac{d}{d t}=\frac{\partial}{\partial t}+\frac{d \theta}{d t} \frac{\partial}{\partial \theta}+\frac{d u}{d t} \frac{\partial}{\partial u}=\frac{\partial}{\partial t}+\Omega_{s}(A) \frac{\partial}{\partial \phi}
$$

where $\phi$ is the phase of the synchrotron oscillations, and $\Omega_{s}(A)$ is synchrotron frequency which can depend on amplitude of the oscillations $A$. Assuming also that the explicit dependence of all the variables on time is given by the factor $\exp (-i \omega t)$, one can rewrite Eq. (6) in the form:

$$
\begin{gathered}
{\left[\left(-i \omega+\Omega_{s} \frac{\partial}{\partial \phi}\right)^{2}+\Omega^{2}(u) Q^{2}(u)\right] X(\theta, u)=} \\
2 \Omega_{0}^{2} Q_{0} \Delta Q(\theta)\left[X(\theta, u)-X_{c}(\theta)\right]+\frac{e G(\theta)}{m \gamma} .
\end{gathered}
$$

Because the betatron frequency is much more than the synchrotron one in practice, this equation can be reduced to a simpler first order form: 


$$
\begin{gathered}
{\left[\omega+\Omega(u) Q(u)+i \Omega_{s} \frac{\partial}{\partial \phi}\right] X(\theta, u) \simeq} \\
\Omega_{0} \Delta Q(\theta)\left[X(\theta, u)-X_{c}(\theta)\right]+\frac{e G(\theta)}{2 m \gamma \Omega_{0} Q_{0}} .
\end{gathered}
$$

Note that the variables $X$ and $X_{c}$ are coupled by the relation:

$$
\int_{-\infty}^{\infty} F(\theta, u) X(\theta, u) d u=X_{c}(\theta) \rho(\theta)
$$

where $F(\theta, u)$ is longitudinal distribution function (actually it depends on amplitude $A(\theta, u)$ ), and $\rho(\theta)$ is corresponding linear beam density:

$$
\rho(\theta)=\int_{-\infty}^{\infty} F(\theta, u) d u
$$

\section{$3 \quad$ Beam field}

The field $G(\theta)$ can be represented in terms of corresponding transverse beam coupling impedance $Z(\omega)$ :

$$
G(\theta)=\frac{i \beta \Omega_{0}}{4 \pi^{2}} \sum_{k} Z\left(\omega+k \Omega_{0}\right) \exp (i k \theta) \int_{0}^{2 \pi} D\left(\theta^{\prime}\right) \exp \left(-i k \theta^{\prime}\right) d \theta^{\prime}
$$

where $D(\theta)$ is linear density of the beam dipole moment. It is more convenient to split the last integral on sum over the bunches, using a definition of $n$-th bunch:

$$
D(\theta)=D\left(\frac{2 \pi n}{h}+\vartheta\right)=D^{(n)}(\vartheta)
$$

where $h$ is RF harmonic number, that is $2 \pi / h$ is space between the bunch centers. In principle, the bunches can have different intensity $N^{(n)}$ and distribution $F^{(n)}$. However, we will use the same normalization:

$$
\int_{-\pi / h}^{\pi / h} \int_{-\infty}^{\infty} F^{(n)}(\vartheta, u) d \vartheta d u=\int_{-\pi / h}^{\pi / h} \rho^{(n)}(\vartheta) d \vartheta=1
$$


Then relation of the beam displacement and dipole moment is

$$
D^{(n)}(\vartheta)=\frac{e N^{(n)} X_{c}^{(n)}(\vartheta) \rho^{(n)}(\vartheta)}{R_{0}}=\frac{e N^{(n)}}{R_{0}} \int_{-\infty}^{\infty} F^{(n)}(\vartheta, u) X^{(n)}(\vartheta, u) d u .
$$

Therefore, Eq. (13) can be written in the form:

$$
\begin{aligned}
G(\theta) & =\frac{i e \beta \Omega_{0}}{4 \pi^{2} R_{0}} \sum_{n=1}^{h} N^{(n)} \sum_{k} Z\left(\omega+k \Omega_{0}\right) \exp (i k[\theta-2 \pi n / h]) \\
& \times \int_{\pi / h}^{\pi / h} \int_{-\infty}^{\infty} F^{(n)}(A(\vartheta, u)) X^{(n)}(\vartheta, u) \exp (-i k \vartheta) d \vartheta d u .
\end{aligned}
$$

Taking into account that in $n$-th bunch

$$
\Delta Q(\theta)=\frac{\Delta Q_{0}^{(n)} \rho^{(n)}(\vartheta)}{\rho_{0}^{(n)}}
$$

it is possible to represent (10) as a set of equations:

$$
\begin{aligned}
& {\left[\omega+\Omega(u) Q(u)+i \Omega_{s} \frac{\partial}{\partial \phi}\right] X^{(n)}(\vartheta, u)-} \\
& \frac{\Omega_{0} \Delta Q_{0}^{(n)}}{\rho_{0}^{(n)}} \int_{-\infty}^{\infty} F^{(n)}\left(\vartheta, u^{\prime}\right)\left[X^{(n)}(\vartheta, u)-X^{(n)}\left(\vartheta, u^{\prime}\right)\right] d u^{\prime}= \\
& \frac{i r_{0} \Omega_{0}}{2 \pi \gamma Q_{0} Z_{0}} \sum_{n^{\prime}=1}^{h} N^{\left(n^{\prime}\right)} \sum_{k} Z\left(\omega+k \Omega_{0}\right) \exp \left(i k\left[\vartheta-2 \pi \frac{n-n^{\prime}}{h}\right]\right) \times \\
& \int_{\pi / h}^{\pi / h} \exp \left(-i k \vartheta^{\prime}\right) d \vartheta^{\prime} \int_{-\infty}^{\infty} F^{\left(n^{\prime}\right)}\left(\vartheta^{\prime}, u^{\prime}\right) X^{\left(n^{\prime}\right)}\left(\vartheta^{\prime}, u^{\prime}\right) d u^{\prime}
\end{aligned}
$$

where $r_{0}=e^{2} / m c^{2}\left(1.535 \times 10^{-16} \mathrm{~cm}\right.$ for protons $)$, and the values with bottom index ' 0 ' are related to the bunch center. 


\section{Rigid modes}

The statement "space charge dominating impedance" means that the righthand part of Eq. (19) is small and can be neglected in first approximation. It is easy to verify that such shortened equation has particular solutions:

$$
X^{(n)}(\vartheta, u)=\mathcal{A}^{(n)} \exp \left(i\left[Q_{0}-\xi / \eta\right] \vartheta\right)
$$

where $\xi=\partial Q / \partial(\ln p)$ and $\eta=-\partial(\ln \Omega) / \partial(\ln p)$ are the machine chromaticity and slippage factor. Set of amplitudes $\mathcal{A}^{(n)}$ forms a collective mode, number of independent modes is coinciding with number of bunches. All these modes are "rigid" in the sense that the coherent displacement does not depend on the particle momentum, as it is seen from Eq. (20). Their

exclusive property is that all of them have the same frequency at $Z=0$, a statement which does not depend on space charge at all: $\omega=-\Omega_{0} Q_{0}$.

To calculate a contribution of the impedance $Z$, it is needed to substitute (20) to the right-hand part of series (19), multiply it on $\exp \left(i\left[\xi / \eta-Q_{0}\right] \vartheta\right)$, and integrate over $\vartheta$. Following series of equations for amplitudes $\mathcal{A}_{n}$ is obtained as a result:

$$
\begin{gathered}
\left(\omega+\Omega_{0} Q_{0}\right) \mathcal{A}^{(n)}=\frac{i r_{0} \Omega_{0}}{2 \pi \gamma Q_{0} Z_{0}} \sum_{k} \mathcal{P}_{k, n} Z\left(\omega+k \Omega_{0}\right) \\
\times \sum_{n^{\prime}=1}^{h} \mathcal{P}_{k, n^{\prime}}^{*} N^{\left(n^{\prime}\right)} \mathcal{A}^{\left(n^{\prime}\right)} \exp \left(2 \pi i k \frac{n^{\prime}-n}{h}\right)
\end{gathered}
$$

where

$$
\mathcal{P}_{k, n}=\int_{\pi / h}^{\pi / h} \rho^{(n)}(\vartheta) \exp \left(i\left[k-Q_{0}+\xi / \eta\right] \vartheta\right) d \vartheta
$$

\section{Symmetric beam}

An ultimate estimation of the instability growth rate can be obtained with help of symmetric beam model, because a removal of several bunches makes the beam more stable [7]. Parameters $N^{(n)}$ and $\mathcal{P}_{k, n}$ do not depend on $n$ in this case, and solution of series (21) is:

$$
\mathcal{A}^{(n)}=\exp \left(\frac{2 \pi i n j}{h}\right)
$$


where $j=0,1, \ldots, h-1$ is index of collective mode (any interval of length $h$ can be used). Because of the symmetry, only harmonics $k=j+h l$ with arbitrary $l$ give a contribution in series (21) resulting in:

$$
\omega+\Omega_{0} Q_{0}=\frac{i r_{0} \Omega_{0} N}{2 \pi \gamma Q_{0} Z_{0}} \sum_{l} Z\left(\Omega_{0} h\left[l+\kappa_{j}\right]\right) S\left(l+\kappa_{j}+\kappa_{\xi}\right)
$$

where $N=h \max \left\{N^{(n)}\right\}$ is total intensity of the symmetrized beam, $\nu$ is a deviation of $Q_{0}$ from the nearest integer $(|\nu|<0.5), \kappa_{j}=(j-\nu) / h, \kappa_{\xi}=$ $\xi / h \eta$, and the parameter

$$
S(\lambda)=\left|\int_{-\pi / h}^{\pi / h} \rho(\theta) \exp (i \lambda h \vartheta) d \vartheta\right|^{2}
$$

should be interpreted as impedance suppression factor. Actually it depends on the product $\lambda B$ where $B$ is bunch-factor, that is a ratio of average beam density to maximal one. With normalization condition (15) taken into account, it is:

$$
B=\frac{h}{2 \pi \rho_{0}} .
$$

In particular, for uniform bunches of length $\vartheta_{0}=2 \pi B / h$ (boxcar beam)

$$
S(\lambda)=\left[\frac{\sin (\pi \lambda B)}{\pi \lambda B}\right]^{2},
$$

and for Gaussian bunches of dispersion $\sigma_{\vartheta}=\sqrt{2 \pi} B / h$ :

$$
S(\lambda)=\exp \left(-\lambda^{2} \sigma^{2} h^{2}\right)=\exp \left(-2 \pi \lambda^{2} B^{2}\right) .
$$

Several examples are considered below.

\subsection{Thick resistive wall instability}

Resistive wall impedance is given by Eq. (1); however, only thick wall is considered in this subsection, that is the assumption $\Omega_{1} \ll \Omega_{0}$ is used. Then it follows from Eq. (24):

$$
\operatorname{Im} \omega=\frac{r_{0} \Omega_{0} N Z^{(r w)}}{2 \pi \gamma Q_{0} Z_{0} \sqrt{|j-\nu|}} f^{(r w)}\left(\kappa_{j}, \kappa_{\xi}\right)
$$




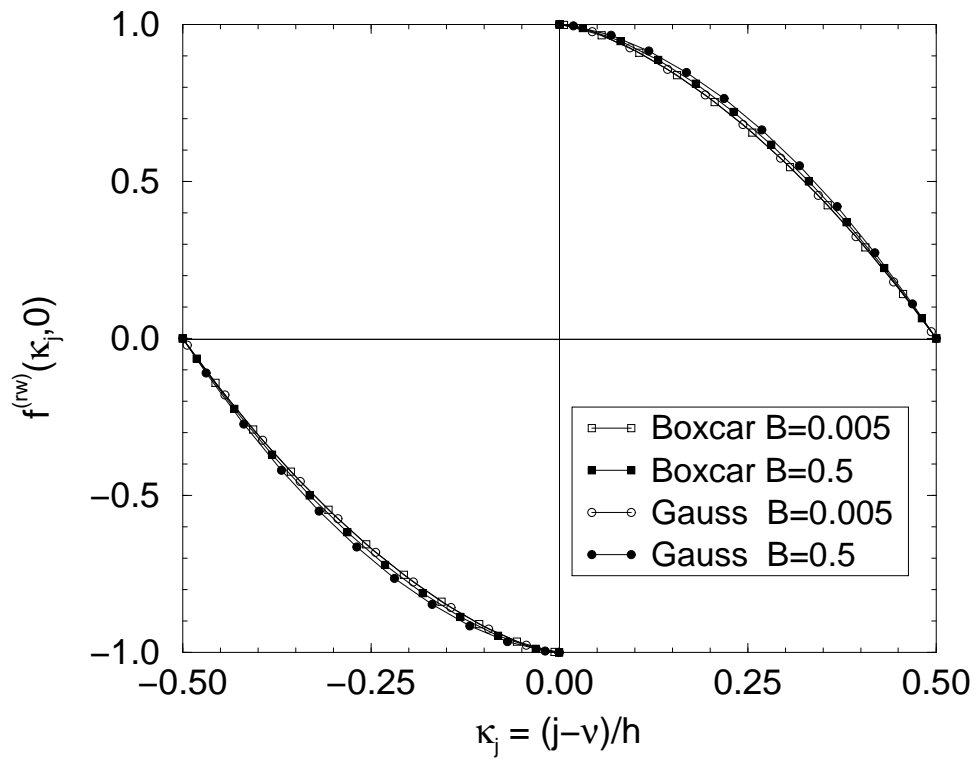

Figure 1: Effect of bunching on instability growth rate at $\xi=0$.

where

$$
f^{(r w)}\left(\kappa_{j}, \kappa_{\xi}\right)=\sqrt{\left|\kappa_{j}\right|} \sum_{l} \frac{\sqrt{\left|l+\kappa_{j}\right|}}{l+\kappa_{j}} S\left(l+\kappa_{j}+\kappa_{\xi}\right) .
$$

First multiplier (fraction) in Eq. (29) coincides with a coasting beam instability growth rate of $j$-th azimuthal harmonic without Landau damping. Therefore, the function $f^{(r w)}$ is the factor describing effect of bunching and chromaticity. Because the right-hand part of (29) is a periodical function of the argument $\kappa_{j}=(j-\nu) / h$ with period 1 , it is sufficient to consider $f^{(r w)}\left(\kappa_{j}, \kappa_{\xi}\right)$ at $\left|\kappa_{j}\right|<1 / 2$ (though this function is not periodical itself). It is plotted in Fig. 1 for boxcar and Gaussian beams at $\xi=0$ and two values of $B$ : 0.005 and 0.5. All the curves presented diverge each from other not more than $\pm 3 \%$, and other realistic distributions (e.g. parabolic) fall in this interval as well. Therefore, the instability without chromaticity does not depend in practice both on bunch-factor and on bunch shape, at least at $B<0.5$. The reason is that only several lower harmonics essentially contribute to the series $(31)$ at $\kappa_{\xi}=0$, and all corresponding $S$-factors are about 1 . The instability is possible only at $\kappa_{j}>0$ i.e. at $j>\nu$, that is 
the beam spectrum contains about $h / 2$ unstable rigid modes. A special case is single bunch when the only mode $j=0$ exists. It can be unstable only at $\nu<0$, that is if the tune $Q_{0}$ is arranged between some half-integer and next integer numbers. This result was obtained first in Ref. [8] at $B=0^{3}$. Note also that the only term $l=0$ essentially contributes to the sum at $\left|\kappa_{j}\right| \ll 1$ resulting in $f^{(r w)}\left(\kappa_{j}, 0\right) \simeq \operatorname{sgn}\left(\kappa_{j}\right)$.

At $\xi \neq 0$, the instability growth rate relatively slightly depends on the bunch shape, but considerably depends on the bunch factor. It is illustrated by Fig. 2 and 3 where the function $f^{(r w)}$ is plotted against $B \kappa_{\xi}=B \xi / h \eta$ for boxcar and Gaussian beams at $B=0.1$ and $B=0.5, \kappa_{j}= \pm 0.1$ and 0.5. It is seen that positive values $\kappa_{\xi}$ are more effective for suppression of the instability, and sufficiently large chromaticity of proper sign kills it at all. The explanation follows from Eq. (28) and (30): such chromaticity attenuates unstable harmonics $l>0$ more than stable harmonics $l<0$. Harmonic $l=0$ gives positive or negative contribution, in dependence on $\operatorname{sgn}\left(\kappa_{j}\right)$. Mainly, its balance with harmonic $l=-\operatorname{sgn}\left(\kappa_{j}\right)$ determines value of $B \kappa_{\xi}$ providing the beam stability. This value is plotted in Fig. 4 against $\kappa_{j}$ at different distributions and bunch-factors. What is interesting, the plot does not depend on the beam intensity.

\subsection{Thin resistive wall instability addition}

Considering thin wall, we will restrict ourself to the case $h \Omega_{0} \gg \Omega_{1}$, which means that skin depth at radio-frequency is much less than the wall thickness. Then inclusion of the last multiplier (coth) in Eq. (1) can change only term $l=0$ in Eq. (30), providing the addition to the instability growth rate (29):

$\Delta \operatorname{Im} \omega=\frac{r_{0} \Omega_{0} N Z^{(r w)} \sqrt{|j-\nu|}}{2 \pi \gamma Q_{0} Z_{0}(j-\nu)} \frac{2 S\left(\kappa_{j}+\kappa_{\xi}\right) \exp \left(-x_{j}\right)\left[\sin x_{j}+\cos x_{j}-\exp \left(-x_{j}\right)\right]}{1-2 \exp \left(-x_{j}\right) \cos x_{j}+\exp \left(-2 x_{j}\right)}$

where $x_{j}=2 \sqrt{|j-\nu| \Omega_{0} / \Omega_{1}}$. This addition does not depend on bunch factor and can be essential only at $x_{j}<\sim 1$. Therefore, at zero chromaticity, it

\footnotetext{
${ }^{3}$ Sometimes the sum over $l$ in Eq. (24) is replaced by an integral over $\omega$ (see for example Ref. [9]). It is unacceptable for resistive wall and many other impedances, because it ignores the dependence on tune, a statement which could be valid at wide-band impedance only.
} 


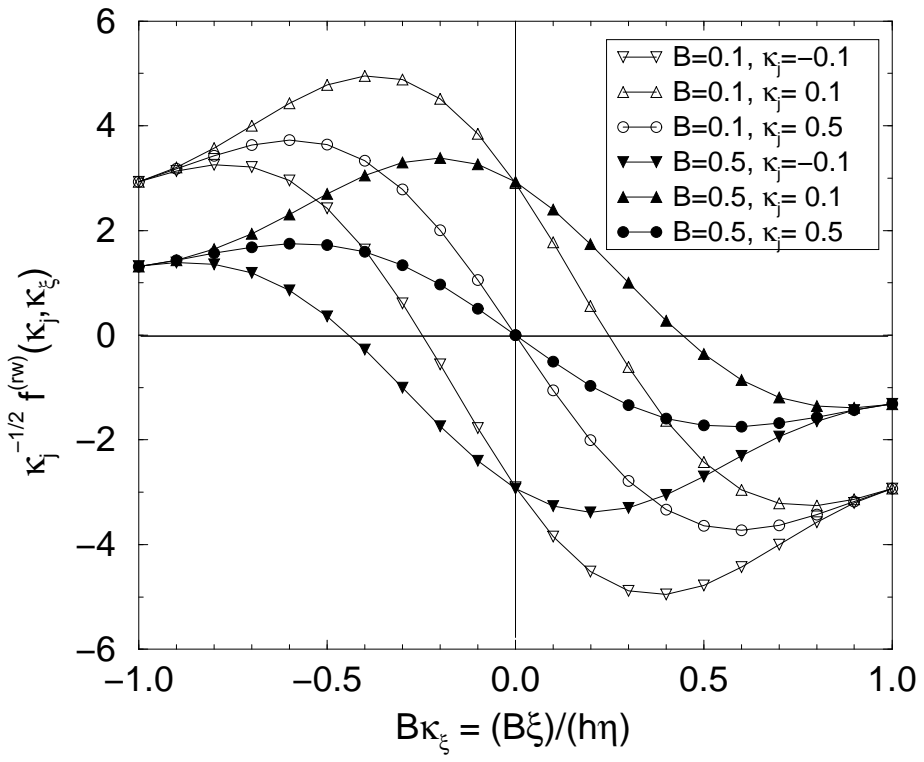

Figure 2: Effect of bunching and chromaticity on the instability growth rate (Boxcar beam).

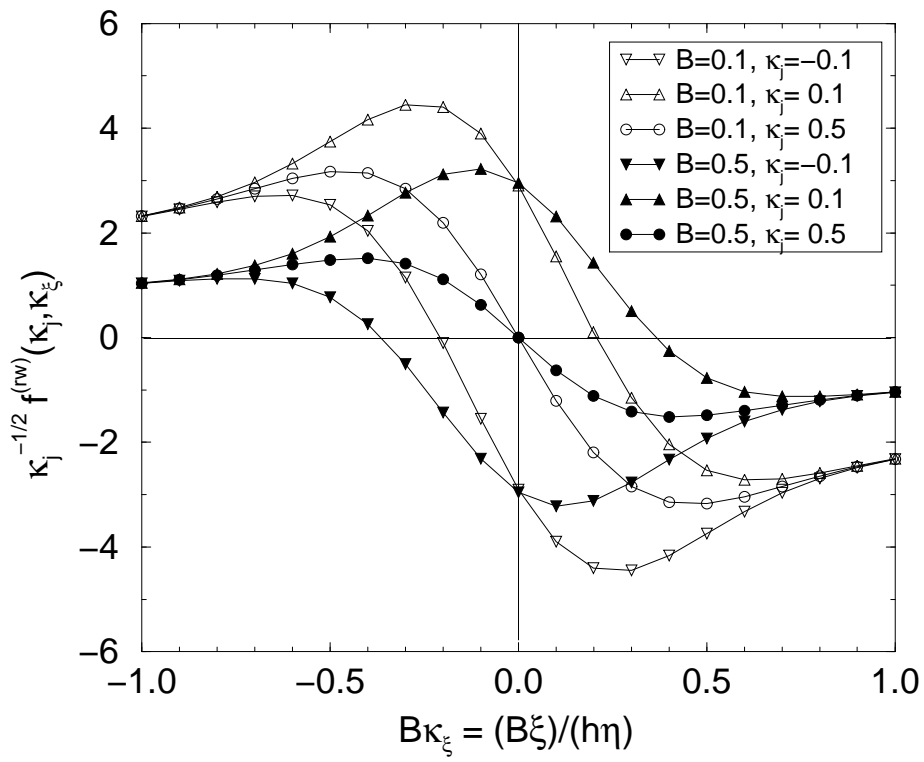

Figure 3: Effect of bunching and chromaticity on the instability growth rate (Gaussian beam). 


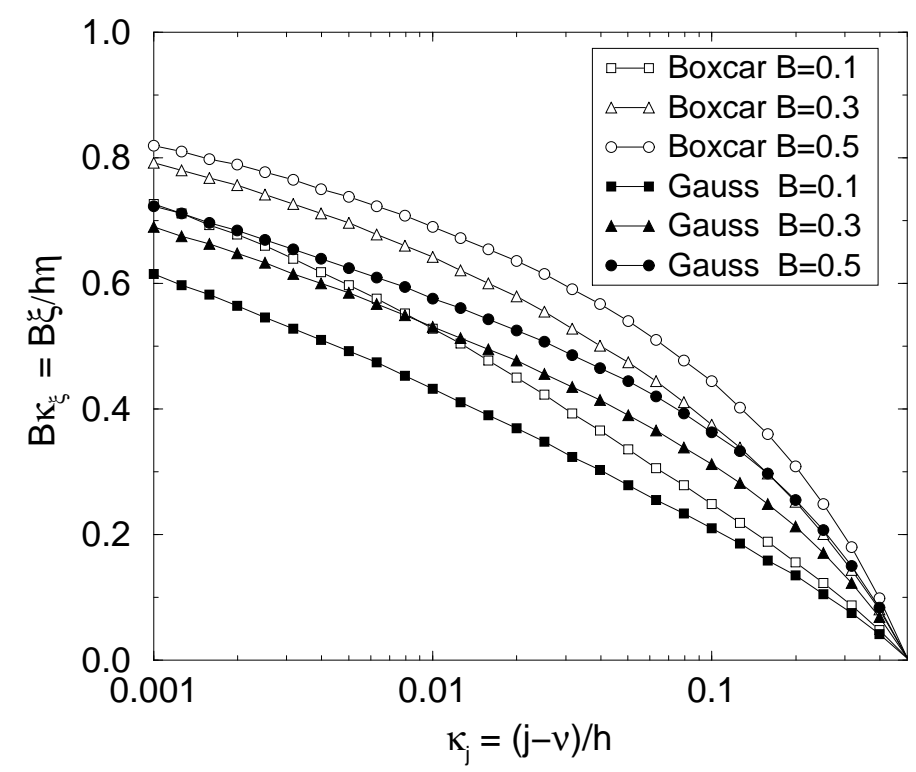

Figure 4: Normalized threshold chromaticity of $j$-th mode in dependence on bunch-factor.

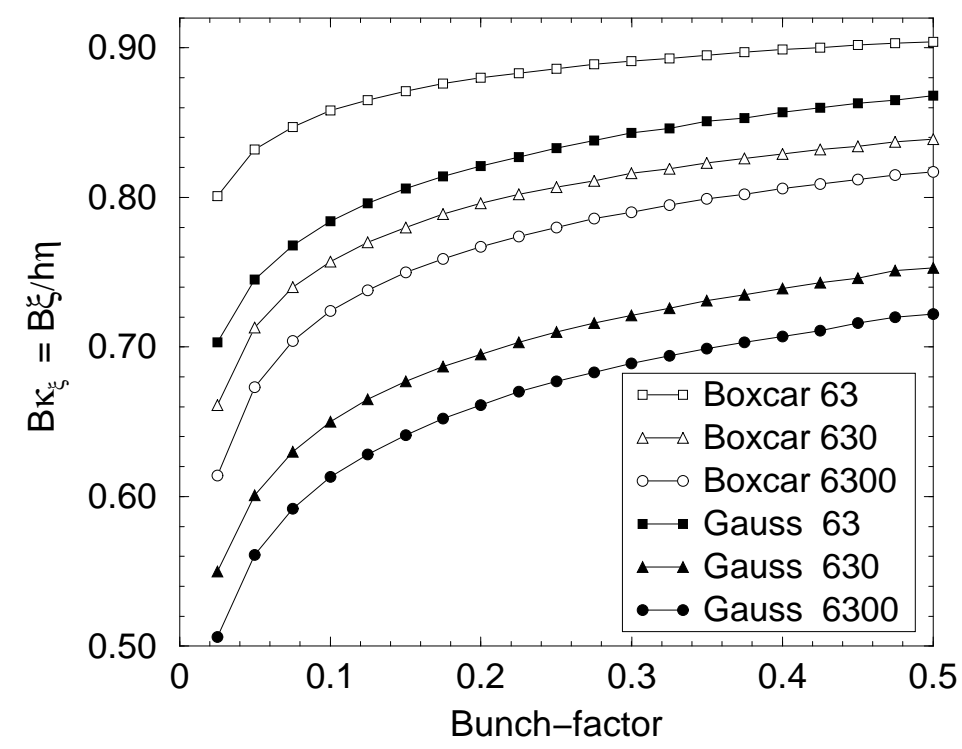

Figure 5: Normalized threshold chromaticity against bunch-factor at $(j-$ $n u) / h=0.001$ and different $h \Omega_{0} / \Omega_{1}$. 
simply increases growth rate of the lower unstable modes, both at coasting and bunched beams. Another effect is a strengthening of higher unstable modes due to more contribution of harmonic $l=0$ at nonzero chromaticity. As a result, more chromaticity is required to suppress the instability. It is illustrated by Fig. 5 where threshold value of $B \xi / h \eta$ is plotted against bunch factor at different ratio $h \Omega_{0} / \Omega_{1}$ (this parameter is proportional to the wall thickness being about 630 at the Recycler).

\subsection{Instability damper}

Let us consider the impedance

$$
Z(\omega)=-Z^{(d m)}\left\{\begin{array}{lll}
1 & \text { at } & |\omega|<h \Omega_{0} / 2 \\
0 & \text { at } & |\omega|>h \Omega_{0} / 2
\end{array}\right.
$$

which is a simplest model of an instability damper with high order low pass filter. The only term $l=0$ remains in Eq. (24) in this case, resulting in:

$$
\operatorname{Im} \omega=-\frac{r_{0} \Omega_{0} N Z^{(d m)}}{2 \pi \gamma Q_{0} Z_{0}} S\left(\frac{j-\nu+\xi / \eta}{h}\right) .
$$

Thus, the damper attenuates the instability growth rate of all rigid modes. One can see that this value falls slower than resistive wall contribution, when the mode index $j$ is increasing. Therefore, suppression of the lowest unstable resistive wall mode assures the suppression of all rigid modes, resulting in the stability condition:

$$
Z^{(d m)}>\frac{Z^{(r w)}}{\sqrt{j-\nu}} \frac{1+2 \exp \left(-x_{j}\right) \sin \left(x_{j}\right)-\exp \left(-2 x_{j}\right)}{1-2 \exp \left(-x_{j}\right) \cos \left(x_{j}\right)+\exp \left(-2 x_{j}\right)}
$$

which should be satisfied at any $j>\nu$ (notation of Eq. (31) is used in the last factor which describes the wall thickness effect). The condition does not include chromaticity at all, because it equally suppresses both resistive wall and damper effects.

It is interesting to compare this result with the case of ultimately wideband damper when $Z=-Z^{(d m)}$ independently on frequency. Then all harmonics $l$ contribute to sum (24) which is calculated analytically resulting in:

$$
\operatorname{Im} \omega=-\frac{r_{0} \Omega_{0} N Z^{(d m)}}{2 \pi \gamma Q_{0} Z_{0} B} \int \frac{\rho^{2}(\theta)}{\rho(0)} d \theta
$$


where normalization condition (15) is used. The last integral only slightly depends on the bunch shape being: (i) 1 for uniform bunch, (ii) $4 / 5$ for parabolic bunch, (iii) $1 / \sqrt{2}$ for Gaussian bunch. Factor $B$ in the denominator means that the damping effect depends the linear density. Another distinction is that effect of the wide-band damper is not attenuated by chromaticity.

\section{Recycler application}

Maximal intensity of the Recycler is expected to be as large as $5.3 \times 10^{13}$ protons in second stage of the Proton Plan [2]. However, only $6 / 7$ of the ring will be filled, that is $N=6.2 \times 10^{13}$ and $h=588$ should be substituted in Eq. (30) and (31) to get the ultimate (probably over) estimation of the instability. We will use resistive wall impedance $(1)$ at $Z^{(r w)}=22 \mathrm{MOhm} / \mathrm{m}$, a value which relates to vertical oscillations. Other parameters needed: $\Omega_{0} / 2 \pi=89.9 \mathrm{kHz}$, $\Omega_{1} / 2 \pi=83 \mathrm{kHz}, \gamma=9.53, \eta=-0.0085, Q_{0}=24.415^{4}$. Then Eq. (29)-(31) give the instability growth rate of the lowest mode $j=1$

$$
\operatorname{Im} \omega=S\left(\frac{0.585+\xi / \eta}{588}\right) \times 3.8 \mathrm{~ms}^{-1} .
$$

Corresponding instability growth time is about $0.3 \mathrm{~ms}$ at $\xi=0$. Total life time of proton beam in the Recycler is expected to be about $1.5 \mathrm{~s}$ including $0.25 \mathrm{~s}$ at maximal intensity. Therefore, the growth rate should be decreased at least by factor $10^{3}$ to ensure the beam stability.

There are two ways to reach this, both resulting in total suppression of the rigid modes. First of them is a use of chromaticity which required value can be obtained from Fig. 5 at $(j-\nu) / h \simeq 0.001$ and $h \Omega_{0} / \Omega_{1} \simeq 760$. Because required chromaticity decreases when bunch factor increases, we take $B=$ $0.4-0.5$ as a reasonable estimation. Then required chromaticity is -7 to -10 in dependence on bunch shape (smooth distribution like Gaussian is better).

It is unclear now what chromaticity will be acceptable at new conditions (nominal value $\xi=-2$ [1]). Therefore another possibility should be considered as well which is $26 \mathrm{MHz}$ damper with impedance $Z^{(d m)}>40 \mathrm{MOhm} / \mathrm{m}$.

\footnotetext{
${ }^{4}$ It is a nominal tune. The result changes but little at other tune in use
} 
If the damper includes single pickup and kicker connected by corresponding feedback circuit, the relation should be satisfied to provide the required impedance:

$$
\frac{\alpha}{J Y_{c}}>4.7 \frac{\mu \mathrm{rad}}{\mathrm{A}-\mathrm{mm}}
$$

where $J$ is the beam current, $Y_{c}$ is its vertical offset in the pickup, and $\alpha$ is corresponding angular deviation produced by the kicker. Two times weaker damper is required for horizontal direction.

\section{Discussion: other modes}

Series (21) does not include characteristics of synchrotron motion at all, such as shape of potential well, frequency, etc. Moreover, its solutions do not depend on space charge as well. One could think on these grounds that the rigid mode instability is a universal effect inherent in all bunched beams. However, this statement needs further consideration because the rigid modes are actually approximate solutions of Eq. (19) at small $Z$, applicability of which depends on above mentioned factors. In principle, a coupling with other modes can change characteristics of the rigid modes or even destroy them.

An example of similar behavior is a bunch in a square potential well. As it was shown in Ref. [10], all its eigenmodes are governed by impedance $Z$ and do not depend on space charge. This conclusion can be obtained from Eq. (10) as well, taking into account that $\Delta Q=$ const and $\theta \propto|\phi|$ in this case. As a result, all eigenfrequencies of the bunch are identical at $G=0$, the rigid mode does not stand out and must not be considered separately from others. Rather close result can be obtained for parabolic potential well when the boxcar model is used, because $\Delta Q=$ const in this case also.

However, the last example demonstrates only that the boxcar beam should not be taken as a realistic model at analysis of the problem. Following consideration does not pretend to rigour and completeness, and is proposed only to support applicability of the theory to the Recycler.

First of all it is necessary to note that space charge tune shift really spreads from 0 to $\Omega_{0} \Delta Q_{0}$, if the bunch is in parabolic potential well. The frequency averaged on period of synchrotron oscillations has the spread $\sim$ $\Omega_{0} \Delta Q_{0} / 2$, and one can expect that the same is true for frequencies of all the 
eigenmodes except the rigid ones. If so, a slot between the rigid and other frequencies can be expected in the spectrum. Its width should be a noticeable part of space charge tune shift, and additional impedance comparable with the space charge contribution would be required to overcome it. However, as it was shown in the Sec. 1, space charge impedance dominates in the Recycler, that is this way to disturb the rigid mode looks unlikely.

Incorporation of synchrotron frequency increases spread of the eigenfrequencies and could decrease the mentioned slot. There is an opinion that fast head-tail instability appears when the synchrotron tune becomes comparable to the betatron tune shift [6]. Still more frequency creates the slot again, but by another mechanism [5]. However, expected synchrotron frequency does not exceed $1 \mathrm{kHz}$ in the Recycler, that is an order of magnitude less than the space charge tune shift. Thus, there are no reasons to think that the coupling can seriously affect the rigid modes in the Recycler, including instability growth rate and conditions of its suppression.

From this point of view, either chromaticity $-\xi \simeq 7-10$ or $26 \mathrm{MHz}$ damper is necessary condition of the Recycler beam stability. However, it is unclear yet whether this condition is sufficient, because other than rigid modes could be unstable as well. The following reasonings let us to think that they are more stable, at least.

There is a spread of incoherent betatron frequency in the beam due to modulation of the space charge density along the bunch. In our case, the spread exceeds the instability growth rate about on order of magnitude. It is known that similar spread produces Landau damping of a coherent oscillations, if their frequency falls into the incoherent spectrum area. That the statement is valid for the space charge produces spread, it was shown in principle in papers [5]-[6]. The rigid modes are, probably, the only case when the coherent frequency is out of this area, and the beam is not subjected to this kind of the Landau damping. Therefore, one can believe that all other modes are not so dangerous, and proposed here stability conditions are not only necessary but also sufficient. 


\section{Conclusion}

It is shown in the paper that, at space charge dominating impedance, there are the modes of bunch oscillations whose shape and frequency do not depend on the intensity at all ("rigid mode"). For a multi-bunch beam, the rigid oscillations of the bunches combine a set of collective modes which differ by phase advance of neighboring bunches. Some of them become unstable under the action of different from the space charge collective forces produced by resistive wall, for example. The instability can be suppressed either by chromaticity or with help of the instability damper. The damper bandwidth should be at least a half of the accelerating frequency to ensure the suppression of all unstable collective modes.

The mentioned collective forces are responsible also for coupling of the rigid and other modes. However, the coupling is actually important only if the space charge tune shift is insufficiently spread, because the beam spectrum is more or less degenerated in this case. The rigid modes are not standing out and must not be considered separately from others at such conditions. A beam in a rectangular potential well or boxcar model applied at any well are typical examples of this.

However, actually the rigid modes differ greatly from others in a parabolic potential well, a statement which refers both to the modes configuration and frequency. The coupling is less important and its neglect should not lead to essential errors in this case. Furthermore, there are reasons to think that the collective rigid modes are most unstable at such conditions, and their suppression ensures a comprehensive transverse beam stability.

For the Fermilab Recycler, the theory predicts resistive wall instability growth rate not more than $1 /(0.3 \mathrm{~ms})$ at the beam intensity $5.3 \times 10^{13}$ in $\sim 500$ bunches (2nd stage of the Proton Plan). The instability can be suppressed by chromaticity from -7 to -10 in dependence on the bunch shape and length. Another possibility considered is the instability damper with $26 \mathrm{MHz}$ bandwidth. 


\section{References}

[1] Gerry Jackson, "The Fermilab Recycler Ring Technical Design Report", Fermilab-TM-1991 (1996).

[2] D. McGinnis, "A 2 Megawatt Multi-Stage Proton Accumulator", beamdocs.fnal.gov/DocDB/0017/001782/007/APS_doc2.pdf (2005).

[3] Martin Hu, James L. Crisp "Fermilab Recycler Damper Requirements and Initial Design", in Proceedings of the 2005 Particle Accelerator Conference, Knoxwill, TN, p.1239 (2005).

[4] V. Balbekov, "Space Charge Impedance and Transverse Beam Instability", Fermilab-FN-0782-AD (2006)

[5] V. I. Balbekov, "Influence of Coulomb shift of betatron frequencies on the stability of coherent oscillations of a circulating bunch", Sov. Phys. Tech. Phys., Vol. 21, No. 7, p.837 (1976).

[6] M. Blaskiewicz, "Fast head-tail instability with space charge", Phys. Rev. ST Accel. Beams 1, 044201 (1998).

[7] V. I. Balbekov, S. V. Ivanov, "Longitudinal instability of azimuthallynonsymmetric beam in a synchrotron", IHEP-89-125 (1989), /accelconf.web.cern.ch/AccelConf/p89/PDF/PAC1989-1400.PDF.

[8] N. Dikansky and A. Skrinsky, Atomnaya Energiya 21, p.176 (1966) (in Russian).

[9] "Handbook of accelerator Physics and Engineering", Edited by A. Chao and M. Tigner, World Scientific 1998, p. 119.

[10] V. Balbekov, "Transverse coherent instability of a bunch on a rectangular potential well", Phys. Rev. ST Accel. Beams 9, 064401 (2006). 


\section{$9 \quad$ Appendix}

It can be shown that, for a beam in a smooth pipe, the coefficient $g$ of Eq. (1) is:

$$
g=\frac{b_{y}^{3}}{16 \pi} \oint\left(\frac{\partial \Phi}{\partial n}\right)^{2} d s
$$

where the integration of the normal derivative is performed over the pipe boundary in plane $x y$. Being zero on this boundary, potential $\Phi$ should satisfy the equation:

$$
\frac{\partial^{2} \Phi}{\partial x^{2}}+\frac{\partial^{2} \Phi}{\partial x^{2}}=-4 \pi \frac{\partial f}{\partial \xi}
$$

where $f(x, y)$ is normalized beam density, and $\xi=x$ or $y$ in dependence on direction of oscillations considered. The coefficients $g_{x, y}$ is plotted below for a thin beam located in the center of an elliptic pipe of semi-axes $b_{x}, b_{y}$. The extreme values are: $g_{x}=g_{y}=1$ in a round pipe, and $g_{x}=\pi^{2} / 24, g_{y}=$ $\pi^{2} / 12$ for two parallel plates.

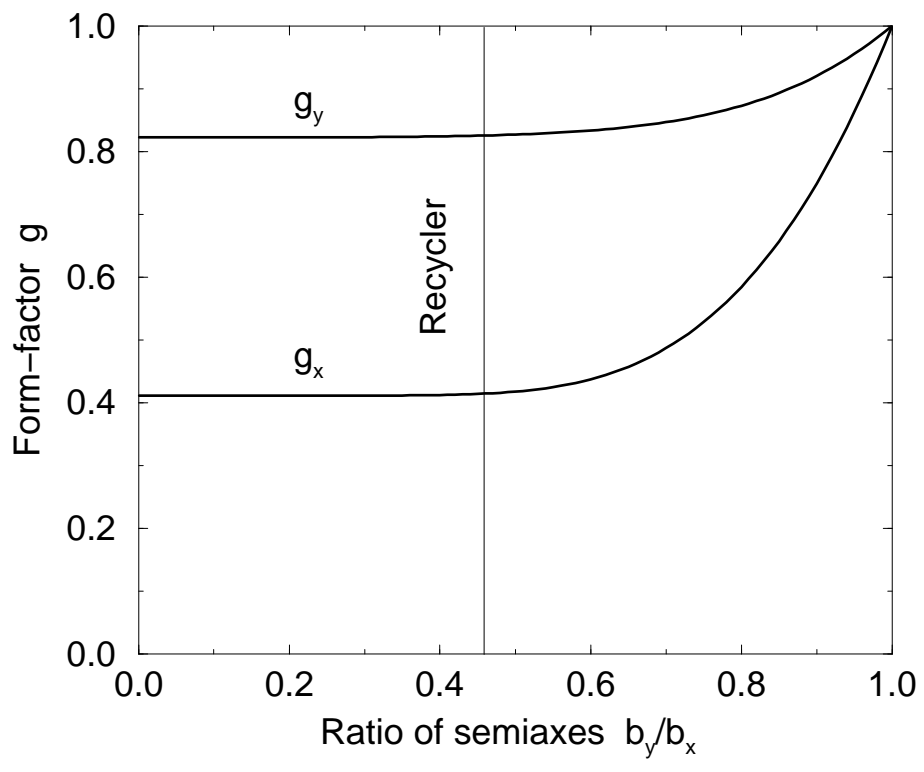

